\title{
In search of advice
}

\author{
We introduce an advisory panel to develop content on the relationship between technology and society.
}

M odern society faces a range of challenges to maintain or improve its relationship with the environment and to reduce inequalities within itself. Science and technology have an essential role to play in addressing these challenges. We need only think, for example, about the development of sustainable and renewable energy sources, the exploration of practical solutions to contain global warming, the search for cures for widespread diseases, or the advances in information and communication technology to improve education in developing countries.

Over the last few years, at Nature Nanotechnology we have paid increasing attention to the ways in which nanomaterials can contribute to addressing societal needs and we are committed to keep doing so in the future. We are also conscious, however, that scientific and technological progress is only part of the solution, albeit an essential one. Especially for an emerging technology, facilitating its positive impact on society requires answering a range of questions. For example, how does the public perceive that technology? Have the material benefits and risks been considered fully? Will the deployment of the technology be environmentally and economically sustainable? Could the technology create more harm than benefits to society, for example in terms of inequalities? Even more fundamentally, is the research itself conducted in a responsible and sustainable way?

We believe that the scientific community should be aware of these issues and take part in the discussion around them. In Nature Nanotechnology we have covered societal aspects of nanotechnology in the past, primarily through the regular contributions of our Thesis writers or through occasional commentaries. We now feel that our commitment to the role of nanotechnology in society should be reflected in more frequent and more structured coverage. With this in mind, from January 2018 our editorial team will be able to count on the support of an external advisory panel to shape and develop our coverage, on such issues as public perception, ethics and philosophy, policy, law and regulations, and sustainability.

The role of the advisory panel will be exclusively to assist our editorial team in commissioning Comments, Perspectives or Reviews. We will also welcome advice on reports in the social-sciences literature that deserve the attention of our audience of researchers in nanoscience and nanotechnology, and that could be featured in Research Highlights, News and Views or Editorials. The panel will, however, will not make decisions on any of the content published in the journal - that remains the remit of the editorial team.

The advisory panel will be in place for an initial period of two years. We will then evaluate the possibility to renew it. At this stage we would like to express our gratitude to the panel members, who have given their time and enthusiastically supported our vision.

Published online: 9 January 2018 https://doi.org/10.1038/s41565-017-0053-3

\section{Bigger and cheaper}

\section{Scalability and cost efficiency should intensify research efforts in DNA nanotechnology.}

t started with M.C. Escher's wood engraving Depth. In the early 1980s,

Nadrian Seeman was looking for a solution to a crystallography problem. The symmetry and the spatial arrangements of the school of fish in Escher's hypnotic panel got him thinking about DNA self-assembly, and the field of DNA nanotechnology was born ${ }^{1}$. Since then, the simple DNA topologies designed by Seeman and colleagues have evolved into complex DNA origami structures that can be programmed to adopt specific shapes and dynamic DNA machines that are capable of molecular sensing ${ }^{2}$.

DNA nanotechnology has so far provided significant fundamental insight into the capabilities of bottom-up molecular assembly, allowing exquisite control over the organization of material and molecules at the nanoscale. But the translation of DNA nanotechnology from an academic concept to a practical tool is still in its infancy. Now a series of papers showcasing micrometre- sized two-dimensional DNA arrays and gigadalton three-dimensional origami structures might move the field a little bit closer to this goal, proving that bigger and more complex architectures can be built with high yields and low error rates ${ }^{3-5}$. Notably, a smart one-pot biotechnological approach published alongside these papers also offers a pragmatic route to cut DNA origami production costs ${ }^{6}$. In this strategy, bacteriophages are programmed to amplify a precisely engineered, self-cleaving DNA template that generates both the scaffold and the staple sequences needed for 'in-phage' self-assembly. The authors of the study predict that the method, scaled up to a typical biotech pilot, will yield kilograms of origami at 0.18 euros per milligram - two to three orders of magnitude lower than the current costs.

Bigger structures are unlikely to be ideal for every practical implementation: with certain in vivo applications, for example, a minimalist approach could allow architectures to be designed that retain complex functions while being simple enough to show low toxicity in the body. But in other cases, such as for energy and photonic applications, large structures are potentially desirable. Moreover, exploring self-assembly at different length scales, from molecular complexes to virus-like vesicles and cell organelles, could unlock new possibilities in the emerging fields of artificial cells and molecular robotics.

Published online: 9 January 2018 https://doi.org/10.1038/s41565-017-0054-2

\footnotetext{
References

1. http://go.nature.com $/ 2 \mathrm{kKrycZ}$

2. Seeman, N. \& Sleiman, H. Nat Rev. Mat. https://doi.org/10.1038/ natrevmats.2017.68 (2017).

3. Tikhomirov, G., Petersen, P. \& Qian, L. Nature 552, 67-71 (2017).

4. Ong, L. L. et al. Nature 552, $72-77$ (2017).

5. Wagenbauer, K. F., Sigl, C. \& Dietz, H. Nature 552, 78-83 (2017).

6. Praetorius, F. et al. Nature 552, 84-87 (2017).
} 